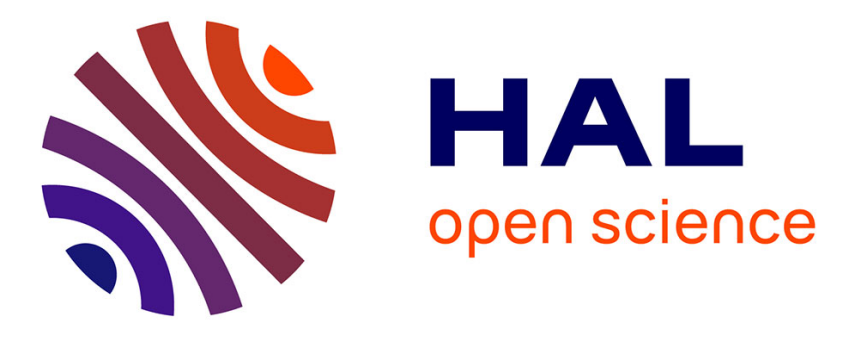

\title{
Shear-Jammed, Fragile, and Steady States in Homogeneously Strained Granular Materials
}

Yiqiu Zhao, Jonathan Barés, Hu Zheng, Joshua E. s. Socolar, Robert P Behringer

\section{- To cite this version:}

Yiqiu Zhao, Jonathan Barés, Hu Zheng, Joshua E. s. Socolar, Robert P Behringer. Shear-Jammed, Fragile, and Steady States in Homogeneously Strained Granular Materials. Physical Review Letters, 2019, 123 (15), pp.158001. 10.1103/PhysRevLett.123.158001 . hal-03346841

\section{HAL Id: hal-03346841 \\ https://hal.science/hal-03346841}

Submitted on 16 Sep 2021

HAL is a multi-disciplinary open access archive for the deposit and dissemination of scientific research documents, whether they are published or not. The documents may come from teaching and research institutions in France or abroad, or from public or private research centers.
L'archive ouverte pluridisciplinaire HAL, est destinée au dépôt et à la diffusion de documents scientifiques de niveau recherche, publiés ou non, émanant des établissements d'enseignement et de recherche français ou étrangers, des laboratoires publics ou privés. 


\title{
Shear-Jammed, Fragile, and Steady States in Homogeneously Strained Granular Materials
}

\author{
Yiqiu Zhao (赵逸秋)①, , $^{*}$ Jonathan Barés, ${ }^{1,2, \dagger}$ Hu Zheng (郑虎), ${ }^{1,3,4,}$ Joshua E. S. Socolar, ${ }^{1, \S}$ and Robert P. Behringer ${ }^{1, \|}$ \\ ${ }^{1}$ Department of Physics \& Center for Nonlinear and Complex Systems, Duke University, Durham, North Carolina 27708, USA \\ ${ }^{2}$ Laboratoire de Mécanique et Génie Civil, Université de Montpellier, CNRS, Montpellier, 34090, France \\ ${ }^{3}$ Department of Geotechnical Engineering, College of Civil Engineering, Tongji University, Shanghai, 200092, China \\ ${ }^{4}$ School of Earth Science and Engineering, Hohai University, Nanjing, Jiangsu, 211100, China
}

(Received 22 April 2019; revised manuscript received 14 August 2019; published 7 October 2019)

\begin{abstract}
We study the jamming phase diagram of sheared granular material using a novel Couette shear setup with a multiring bottom. The setup uses small basal friction forces to apply a volume-conserving linear shear with no shear band to a granular system composed of frictional photoelastic discs. The setup can generate arbitrarily large shear strain due to its circular geometry, and the shear direction can be reversed, allowing us to measure a feature that distinguishes shear-jammed from fragile states. We report systematic measurements of the stress, strain, and contact network structure at phase boundaries that have been difficult to access by traditional experimental techniques, including the yield stress curve and the jamming curve close to $\phi_{\mathrm{SJ}} \approx 0.75$, the smallest packing fraction supporting a shear-jammed state. We observe fragile states created under large shear strain over a range of $\phi<\phi_{\mathrm{SJ}}$. We also find a transition in the character of the quasistatic steady flow centered around $\phi_{\mathrm{SJ}}$ on the yield curve as a function of packing fraction. Near $\phi_{\mathrm{SJ}}$, the average contact number, fabric anisotropy, and nonrattler fraction all show a change of slope. Above $\phi_{F} \approx 0.7$ the steady flow shows measurable deviations from the basal linear shear profile, and above $\phi_{b} \approx 0.78$ the flow is localized in a shear band.
\end{abstract}

DOI: 10.1103/PhysRevLett.123.158001

When a granular material prepared in a stress-free state is sheared, it can make a transition into a mechanically stable state through a process known as shear jamming [1]. Shear jamming occurs in many different systems, including glasses [2], suspensions [3-8], and dry granular matter with [1,9-13] or without [14-17] friction. In 2011, Bi et al. [1] provided a jamming phase diagram [Fig. 1(a)] that extended the Liu-Nagel framework [18] by including a region of shear-jammed (SJ) states for frictional granular materials at finite shear stress with packing fractions $\phi$ between a critical value $\phi_{\mathrm{SJ}}$ and $\phi_{J}^{0}$, the isotropic jamming packing fraction for frictionless particles. Starting from a stress-free state, applying shear strain $\gamma$ can lead to two different types of jammed states: fragile $(F)$ states that are only stable for compatible loads, and SJ states that are stable to reverse shear $[1,19]$. A minimum shear strain $\gamma_{\mathrm{SJ}}(\phi)$ is needed to create a SJ state for fixed $\phi$. In the past decade, many efforts have focused on explaining the origin of rigidity in sheared granular matter with $\phi$ close to the high packing fraction portion of the jamming curve [the yellow curve in Fig. 1(a)] [1,12,15,20-23]. However, less attention has been paid to other parts of the phase diagram, in particular to the yield stress curve, which is important for the rheology of dense granular flow, or to the jamming curve close to the critical packing fraction $\phi_{\mathrm{SJ}}$, where the relation between the shear strain $\gamma$ and jamming has not been experimentally determined.
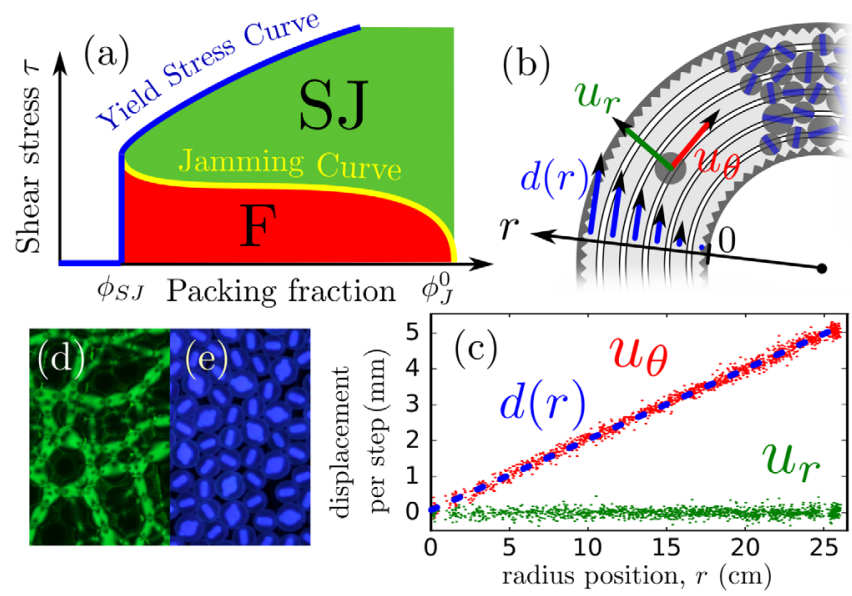

FIG. 1. (a) The jamming phase diagram in the shear stress $\tau$ and packing fraction $\phi$ plane adapted from [24]. Only the $\phi<\phi_{J}^{0}$ part of the diagram is shown. The yield stress curve and the jamming curve are highlighted in blue and yellow, respectively. (b) Schematic of the multiring Couette setup. Twenty-one horizontal concentric rings rotate step wisely to quasistatically shear bidisperse photoelastic discs. For each shear step, the ring at position $r$ rotates by an arc length $d(r)$. The nominal shear strain is defined as $\gamma=d(r) / r$. (c) Particle displacements in radial $\left(u_{r}\right)$ and azimuthal $\left(u_{\theta}\right)$ directions in a shear step for a dilute system $(\phi=0.57)$. The dashed line is the linear basal profile $d(r)$. After each shear step the system is imaged in uv light (e) and in polarized green light $(\mathrm{d})$. 
Experimental measurements of the phase boundaries in the jamming phase diagram are challenging because it is hard to create SJ states without the formation of a shear band and the associated heterogeneities in the packing fraction $\phi$ and strain field [10,25-29]. In 2013, Ren et al. [10] developed a multislat, simple shear setup that avoids shear banding, which revealed a distinction between $F$ and SJ states $[21,23]$. However, their multislat setup had a strain limit ( 60\%) [10], and thus could not access the yield stress curve or the SJ states near $\phi_{\mathrm{SJ}}$, where $\gamma_{\mathrm{SJ}}$ keeps growing as $\phi \rightarrow \phi_{\text {SJ }}^{+}[1,11,15,16]$.

In this Letter, we solve this challenge using a multiring Couette shear setup, which applies a linear shear strain field using basal friction forces to drive the system until it becomes shear jammed. This form of driving may be thought of as a physical implementation of the algorithm used in certain athermal, quasistatic simulations $[20,30]$. With our apparatus, we can also keep shearing the jammed system using boundary racks to measure the yield stress curve. By shearing a layer of photoelastic disks, we for the first time experimentally map out the phase boundaries in the jamming phase diagram close to $\phi_{\mathrm{SJ}}$, including the yield stress curve and the jamming curve. We find that fragile states exist below $\phi_{\mathrm{SJ}}$ that were not included in the traditional phase diagram [1]. Moreover, we find two transitions on the yield stress curve: (i) above $\phi_{F} \approx 0.7$, the steady states no longer deform linearly under shear, and (ii) above $\phi_{b} \approx 0.78$ their deformation field becomes localized. We relate those transitions to the contact network structures.

Experiments. - The experiments are carried out with a novel multiring Couette shear setup shown in Fig. 1(b), which quasistatically and linearly shears a 2D granular medium composed of bidisperse photoelastic discs with friction coefficient 0.9 and diameters 1.59 and $1.27 \mathrm{~cm}$ (denoted as $d$ ) [31]. The ratio of the numbers of big and small particles is $1 / 3$. Particles have reflective paint on their bases to enable reflective photoelasticimetry [34-37]. The total number of particles is varied from 1447 to 2101, which corresponds to $0.56<\phi<0.82$. The Couette setup consists of 21 independently controlled concentric rings. The $1.2 \mathrm{~cm}$ wide rings rotate collectively, providing weak frictional forces to the particles sitting on them. Although essential to perform the linear shear, the magnitude of the basal friction is $\sim 8$ times smaller than the typical contact forces measured in the SJ states on the jamming curve [Fig. 1(a)]. Particles are constrained radially by outer and inner toothed boundaries of radius $r_{\text {out }}=35.5 \mathrm{~cm}$ and $r_{\text {in }}=8.7 \mathrm{~cm}$. The outer boundary rotates with the rings and the inner boundary is fixed.

For each experiment, a stress-free random configuration is prepared. The quasistatic linear shear is then applied in a stepwise manner. For each step, the ring at radial position $r$ rotates through an arc length $d(r)=\gamma r$. The function $d(r)$ sets the "basal profile" and $\gamma$ is called the "shear strain" by analogy with traditional simple shear [10]. We note that $\gamma$ is not the physical shear strain, i.e., the off-diagonal element of the strain tensor, $\varepsilon_{r \theta}=\partial_{r} d(r)-d(r) /\left(r+r_{\text {in }}\right)=$ $\gamma r_{\text {in }} /\left(r+r_{\text {in }}\right)$ [38]. During a rotation step, in which $\delta \gamma=0.6 \%$, the shear rate is $\dot{\gamma} \sim 10^{-3} \mathrm{~s}^{-1}$. After each step, the rings stop for $10 \mathrm{~s}$ to let the system reach a static state. As plotted in Fig. 1(c), for a dilute system, the azimuthal particle displacements $u_{\theta}$ per step follow $d(r)$, and the radial displacements $u_{r}$ fluctuate around zero. No shear band is observed. We apply large forward strains to measure the yield stress curve, and the strain direction is then reversed to distinguish fragile and shear-jammed states.

The system is sequentially lit from the top by circular polarized green light, and from the side by ultraviolet (uv) light [31]. Between two consecutive shear steps, after reaching a static state, the system is imaged (Canon EOS $70 \mathrm{D}, 5472 \times 3648 \mathrm{px}^{2}$ ) through a circular polarizer with uv and polarized lights. Ultraviolet images [Fig. 1(e)] give particle positions. The polarized images [Fig. 1(d)] give stress and contact information. We measure the pressure $P$, defined as the trace of the force moment tensor $[1,10]$, using the averaged squared intensity gradient $[9,10,36,37,39,40]$ of the polarized image [31]. A sheared system must develop a nonzero $P$ to resist finite shear stress $\tau$. We also measure the nonrattler contact number $Z_{\mathrm{nr}}$, defined as the mean contact number among stressed grains $[1,36,41]$, the nonrattler fraction $f_{\mathrm{nr}}$, defined as the number fraction of stressed grains, and the fabric anisotropy $\rho$, defined as the ratio between the difference and the sum of the eigenvalues of the fabric tensor [31]. (See Ref. [36] for a detailed description of the contact detection algorithm.)

Results.-Figures 2(a) and 2(b) show pressure $P$ and nonrattler contact number $Z_{\mathrm{nr}}$ versus shear strain $\gamma$, for typical runs with different $\phi$. For a given $\phi$, after a transient growth regime, both $Z_{\mathrm{nr}}$ and $P$ fluctuate around constant values that define the yield stress curve. We refer the associated stress as the "steady state" stress. We find that $Z_{\text {nr }}$ can be fitted to

$$
Q=Q_{\mathrm{st}}+c e^{-\gamma / \gamma_{c}},
$$

where $Q$ can be $Z_{\mathrm{nr}}, f_{\mathrm{nr}}$, or $1-\rho$, and $Q_{\mathrm{st}}, c$, and $\gamma_{c}$ are fit parameters. An example fit for $Z_{\mathrm{nr}}(\gamma)$ with $\phi=0.76$ is plotted in Fig. 2(b). We find that the steady regime has been reached at $\gamma_{\mathrm{st}} \equiv 3 \gamma_{c}$ for all state variables, where $\gamma_{c}$ is obtained from the fits for $Z_{\mathrm{nr}}$. Figure 2(c) shows $\gamma_{\mathrm{st}}(\phi)$, where a linear fit $\gamma_{\mathrm{st}} \propto\left(\phi-\phi_{0}\right)$ for $\phi>0.72$ gives $\phi_{0}=0.84 \pm 0.02$, close to the frictionless isotropic jamming density [42]. The slope is $-1545 \pm 427(\%)$.

We identify a system as shear jammed if under reverse shear the pressure never drops below the noise threshold $P_{\text {noise }}=0.3 \mathrm{~N} / \mathrm{m}$ [31], which indicates that the system resists the reversed stress rather than simply allowing a reversion to a stress-free (unjammed) state. Figure 2(d) shows the evolution of $P$ during a shear cycle for a system 

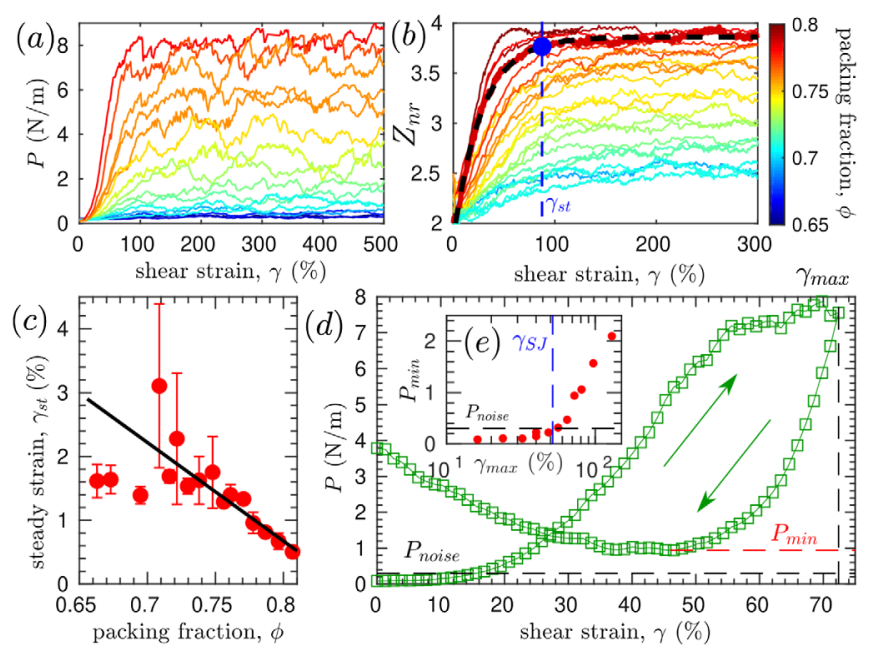

FIG. 2. (a),(b) The pressure $P$ and the nonrattler contact number $Z_{\mathrm{nr}}$ vs $\gamma$ for different $\phi$ during forward shear. The dashed black curve in (b) plots the exponential fit by Eq. (1) for $Z_{\mathrm{nr}}(\gamma)$ with $\phi=0.78$. The blue dashed line shows the $\gamma_{\text {st }}$ value for this run. (c) Strain needed to reach the steady regime $\gamma_{\mathrm{st}}(\phi)$. The black line is a linear fit $\gamma_{\mathrm{st}} \propto\left(\phi-\phi_{0}\right)$ for $\phi>0.72$. (d) $P$ vs $\gamma$ for a typical reverse shear test $(\phi=0.781)$ with forward shear strain $\gamma_{\max }$. (e) The minimum pressure, $P_{\min }$, during the reverse shear vs $\gamma_{\max }$ for $\phi=0.781 . \gamma_{\mathrm{SJ}}$ is the minimum $\gamma_{\max }$ for which $P_{\text {min }}>P_{\text {noise }}=0.3 \mathrm{~N} / \mathrm{m} \mathrm{[31]}$.

with $\phi=0.781$. Figure 2(e) plots the dependence of the minimum pressure $P_{\min }$ during reverse shear on the maximum forward shear strain $\gamma_{\max }$, from which we extract the minimum strain, $\gamma_{\mathrm{SJ}}$, required to create a SJ state. We find no SJ state for $\phi=0.74$ even when $\gamma_{\max } \gg \gamma_{\mathrm{st}}$ [31]. For $\phi=0.75$, we find $\gamma_{\mathrm{SJ}} \approx \gamma_{\mathrm{st}}$. The minimum packing fraction that supports shear jamming must lie between these two values: $\phi_{\mathrm{SJ}}=0.745 \pm 0.005$. Figure 3 (a) plots the relation between $\gamma_{\mathrm{SJ}}$ and $\phi$, which can be fitted using a form suggested in Ref. [15],

$$
\gamma_{\mathrm{SJ}}(\phi)=\gamma_{b}\left[\ln \left(\frac{\phi_{J}^{0}-\phi_{\mathrm{SJ}}}{\phi-\phi_{\mathrm{SJ}}}\right)\right]^{\alpha}
$$

where $\phi_{\mathrm{SJ}}=0.745$ is preset and the fit parameters are $\alpha=0.68 \pm 0.11, \gamma_{b}=64 \pm 6(\%)$, and $\phi_{J}^{0}=0.820 \pm 0.005$.

In this work, fragile $(F)$ states refer to states with nonzero pressure $\left(P>P_{\text {noise }}\right)$ and have $P_{\min }<P_{\text {noise }}$ at some point in the reverse shear process. As shown in Fig. 3(a), we find $\gamma_{F}$, the minimum strain required to create a fragile state, also follows Eq. (2). In this fit, we take $\phi_{J}^{0}=0.82$ from the previous fit, and we determine $\phi_{F}$, the minimum packing fraction for fragile states, from the fit, obtaining $\phi_{F}=$ $0.706 \pm 0.003$ along with $\gamma_{b}=19 \pm 2(\%)$ and $\alpha=$ $0.86 \pm 0.12$. We also note, however, that the divergence predicted by Eq. (2) near $\phi_{\mathrm{SJ}}$ and $\phi_{F}$ is not clearly seen in our data. Below $\phi_{F}$, the steady state pressure falls to a plateau value near the noise level.
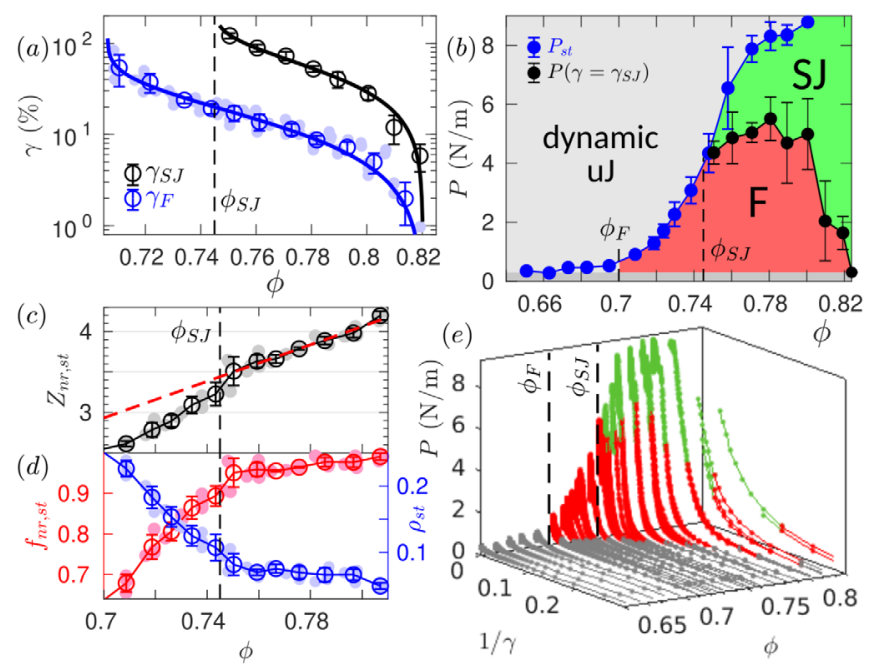

FIG. 3. (a) Strain needed to create fragile state, $\gamma_{F}$ (blue), and SJ state, $\gamma_{\mathrm{SJ}}$ (black). The minimum packing fraction for the SJ states is $\phi_{\mathrm{SJ}} \approx 0.745$. The blue and black solid curves are fitted with Eq. (2). In (a), (c), and (d), solid gray circles are raw data and open circles are averaged data. (b) The jamming phase diagram in the $(P, \phi)$ plane built from our data. The dynamic unjammed $(\mathrm{uJ})$, fragile $(F)$, and shear jammed (SJ) states are separated by the yield stress curve $P_{\mathrm{st}}$ and the jamming curve $P\left(\gamma=\gamma_{\mathrm{SJ}}\right)$. The dark gray region below the noise level $0.3 \mathrm{~N} / \mathrm{m}$ indicates static unjammed states. $\phi_{F}$ is the minimum packing fraction for fragile states. (c),(d) The steady state nonrattler contact number $Z_{\mathrm{nr}, \mathrm{st}}$, nonrattler fraction $f_{\mathrm{nr}, \mathrm{st}}$ and fabric anisotropy $\rho_{\mathrm{st}}$ obtained from Eq. (1). Note the change in slope near $\phi_{\mathrm{SJ}}$ in all three cases. The red dashed line in (c) shows a linear fit using data above $\phi_{\mathrm{SJ}}$. (e) Surface plot of all static states measured during forward shear experiments in the space of $P, \phi$, and inverted strain $1 / \gamma$ space. Smooth curves join states accessed in a single run. States are labeled using the same color code as in (b).

Figure 3(b) shows the experimentally constructed jamming phase diagram in the $(P, \phi)$ space. The yield stress curve is the $P_{\mathrm{st}}(\phi)$ curve, showing the average steady state pressure for each $\phi . P_{\mathrm{st}}$ increases monotonically from $\phi_{F}$ and appears to have an inflection point at $\phi_{\mathrm{SJ}}$. However, for the steady states above $\phi \approx 0.78$, the pressure of some particles becomes so large that their photoelastic fringes cannot be resolved, likely leading to artificially low pressure measurements. $P_{\mathrm{st}}(\phi)$ also separates SJ states and the dynamic unjammed states, which have nonzero shear rates. The jamming curve is also plotted as the $P\left(\phi, \gamma_{\mathrm{SJ}}\right)$ curve, which consists of the pressure value for each $\phi$ at the jamming strain $\gamma_{\mathrm{SJ}}$. The gray region below $P_{\text {noise }}$ refers to the static unjammed states without measurable stress. Figure 3(e) extends 3(b) by including the inverted strain axis and plots all the static states measured during the forward shear process in the $(P, \phi, 1 / \gamma)$ space, highlighting their dependence on the driving strain $\gamma$. A state is labeled SJ when the shear strain exceeds $\gamma_{\mathrm{SJ}}$ determined using Eq. (2). All static SJ (green), $F$ (red), and unjammed (gray) states lie approximately on a smooth surface in the 3D space. 


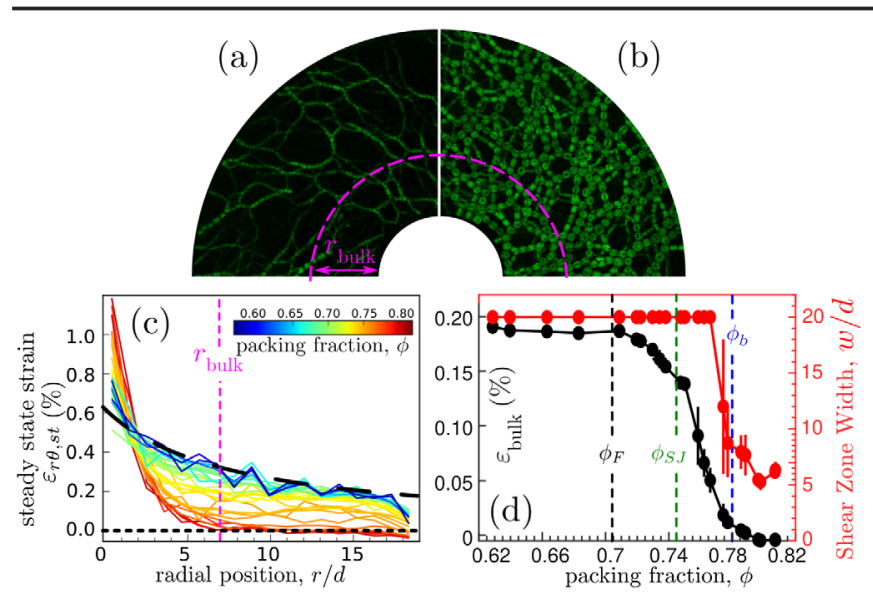

FIG. 4. (a),(b) Polarized images showing force networks of typical steady states with packing fraction 0.72 and 0.78 . (c) Physical shear strain per step averaged over steady states, $\varepsilon_{r \theta, \mathrm{st}}(r)$ for different $\phi$, labeled by the color bar. The black dashed curve shows the basal profile with linear nominal strain $\gamma \cdot r_{\text {bulk }}=$ $7 d$ is where $\varepsilon_{r \theta \text {,st }}$ vanishes for $\phi>\phi_{b}$. (d) $\varepsilon_{\text {bulk }}$, defined as the averaged $\varepsilon_{r \theta \text {,st }}$ for $r>r_{\text {bulk }}$, drops at $\phi_{\mathrm{SJ}}$ and vanishes at $\phi_{b}$. The same figure plots the width of shear zone $w$, defined as the range of $r$ that $\epsilon_{r \theta \text {,st }}$ is nonzero. $w(\phi)$ drops to $r_{\text {bulk }}$ at $\phi \approx \phi_{b}$.

To quantify the contact network structure on the yield stress curve, we measure $Z_{\mathrm{nr}, \mathrm{st}}, f_{\mathrm{nr}, \mathrm{st}}$, and $\rho_{\mathrm{st}}$, which are obtained from fits to the form of Eq. (1). Figures 3(c) and 3(d) show a change in slope in all three state variables at a packing fraction slightly above $\phi_{\mathrm{SJ}}$. The red dashed line in Fig. 3(c) is the linear fit using data with $\phi>\phi_{\mathrm{SJ}}$, which highlights the change in behavior at $\phi_{\mathrm{SJ}}$. Figures $4(\mathrm{a})$ and 4(b) show two polarized images taken from the steady regime with packing fractions 0.72 and 0.78 , showing typical force network in $F$ and SJ states.

When the system is shear jammed, the basal friction becomes unimportant, and the particle displacement field deviates from the basal profile. Based on the azimuthal displacement field per shear step averaged over the steady states, $u_{\theta, \text { st }}(r)$, we calculate the off-diagonal element of the strain tensor $\varepsilon_{r \theta, \mathrm{st}}(r)=\partial_{r} u_{\theta, \mathrm{st}}(r)-u_{\theta, \mathrm{st}}(r) /\left(r+r_{\text {in }}\right)$ [38], which gives the mean physical shear strain field for steady states [Fig. 4(c)]. We also measure the width of the shear zone $w$, which is the $r$ value beyond which $\varepsilon_{r \theta \text {,st }}$ becomes smaller than the noise level $0.02 \%$. Figure 4(d) shows $w(\phi)$ (in red), which jumps discontinuously near $\phi_{b} \approx 0.78$, below which $w=r_{\text {out }}-r_{\text {in }} \approx 20 d$. Above $\phi_{b}, w \approx 7 d$, denoted $r_{\text {bulk }}$ in Fig. 4(c). The local packing fraction in this shear band is also smaller than the global value. The part of the system with $r>r_{\text {bulk }}$ just rotates as a solid with the moving outer boundary in the steady states for $\phi>\phi_{b}$. We also calculate $\varepsilon_{\text {bulk }}$, which is the averaged $\varepsilon_{r \theta \text {,st }}$ for $r>r_{\text {bulk }}$. Figure 4(d) shows $\varepsilon_{\text {bulk }}$ starts to drop at $\phi_{F}$ and becomes zero near $\phi_{b}$.

Concluding discussion.-We set up a multiring Couette device that uses a small basal friction to drive a 2D granular medium in a way that maintains a linear shear strain profile until the system becomes jammed, allowing us to probe the jamming transition close to $\phi_{\mathrm{SJ}}$. The setup subsequently shears the jammed system using the boundary racks, allowing a study of the yield stress curve for a wide range of packing fractions. Finally, reversing the direction of the drive allows us to distinguish SJ from $F$ states.

We systematically measured the phase boundaries in the jamming phase diagram, including close to $\phi_{\mathrm{SJ}}$, leading to the following key observations. (i) In our system $\phi_{\mathrm{SJ}} \approx 0.75$, whose value may depend on the friction coefficient $\mu$, polydispersity, and particle shape, though we expect the qualitative features of the jamming phase diagram to be the same. (ii) The SJ strain $\gamma_{\mathrm{SJ}}$ is well fit by a stretched logarithmic function of $\phi$. The measured exponent $\alpha=0.68 \pm 0.11$ is in quantitative agreement with the exponent $\alpha=1 / 1.37 \approx 0.73$ measured from the simulation of the sheared 3D frictionless soft spheres [15]. The same form, but with $\alpha=1$, has also been observed in experiments on shear-thickening suspensions [5]. (iii) We observe fragile states below $\phi_{\mathrm{SJ}}$, which are not included in the traditional phase diagram [1]. In our system, small basal friction forces and particle deformability may be crucial for stabilizing the fragile force network. (iv) On the yield stress curve, for increasing packing fraction, we find that $P_{\text {st }}$ has an inflection point at $\phi_{\mathrm{SJ}}$ and that $Z_{\mathrm{nr}, \mathrm{st}}, \rho_{\mathrm{st}}$, and $f_{\mathrm{nr}, \mathrm{st}}$ all show a change of slope near $\phi_{\mathrm{SJ}}$, suggesting a physical transition in the nature of the steady states.

We also find that the quasistatic steady flow field changes from the nonlocalized basal profile for systems with $\phi<\phi_{F} \approx 0.7$ to a localized shear band for $\phi>\phi_{b} \approx 0.78$, where $\phi_{F}<\phi_{\mathrm{SJ}}<\phi_{b}$. The coexistence of a solid and fluid phase in slowly sheared dense granular matter has been reported in many systems [25,27,28, 43-46]. In this Letter, we characterize the contact network associated with the different quasistatic steady flow regimes. When $\phi=\phi_{b}$, the steady states have $\rho_{\text {st }} \approx 0.05$ and $f_{\text {nr,st }} \approx 1$, showing a nearly isotropic, fully percolated contact network. Notably, $\phi_{b} \approx \phi_{J}^{\mu}$ with $\mu \approx 0.9$, where $\phi_{J}^{\mu}$ is the isotropic jamming packing fraction with friction coefficient $\mu$ [47]. We also note that $Z_{\mathrm{nr}, \mathrm{st}}\left(\phi_{\mathrm{SJ}}\right) \approx 3.4$, similar to the mean contact number observed when a strong force network percolates in both principal directions in biaxial experiments [1], and $Z_{\mathrm{nr}, \mathrm{st}}\left(\phi_{b}\right) \approx 3.9$, close to the isostatic value for ideal frictionless disks $[42,48]$.

The results suggest several directions for further study. First, our shear device can generate other basal profiles [35] to study how shear jamming affects the granular rheology for shear fields found in real world applications. Second, the setup can create a controlled shear band, providing a new technique to study the generation and evolution of shear bands in dense granular flow.

We thank Bulbul Charkraborty, Dong Wang, Mark D. Shattuck, Karen E. Daniels, Stefan Luding, Dapeng Bi, 
Hisao Hayakawa, and Michael Rubinstein for fruitful discussions. Special thanks to Dong Wang, Yuchen Zhao, Bernie Jelinek, and Richard Nappi for technical support. This work was supported by NSF-DMR1206351, NSFDMS1248071, and NASA NNX15AD38G. H.Z. also received support from NSFC 41672256 and NSFC (Jiangsu) BK20180074.

*yiqiu.zhao@duke.edu

†b@jonathan-bares.eu

tjzhenghu@gmail.com

\$socolar@phy.duke.edu

"Deceased, July 2018.

[1] D. Bi, J. Zhang, B. Chakraborty, and R. P. Behringer, Nature (London) 480, 355 (2011).

[2] P. Urbani and F. Zamponi, Phys. Rev. Lett. 118, 038001 (2017).

[3] E. Han, I. R. Peters, and H. M. Jaeger, Nat. Commun. 7, 12243 (2016).

[4] S. Majumdar, I. R. Peters, E. Han, and H. M. Jaeger, Phys. Rev. E 95, 012603 (2017).

[5] E. Han, M. Wyart, I. R. Peters, and H. M. Jaeger, Phys. Rev. Fluids 3, 073301 (2018).

[6] N. M. James, E. Han, R. A. L. de la Cruz, J. Jureller, and H. M. Jaeger, Nat. Mater. 17, 965 (2018).

[7] D. Z. Chen, H. Zheng, D. Wang, and R. P. Behringer, Nat. Commun. 10, 1283 (2019).

[8] R. Seto, A. Singh, B. Chakraborty, M. M. Denn, and J. F. Morris, Granular Matter 21, 82 (2019).

[9] D. Howell, R. P. Behringer, and C. Veje, Phys. Rev. Lett. 82, 5241 (1999).

[10] J. Ren, J. A. Dijksman, and R. P. Behringer, Phys. Rev. Lett. 110, 018302 (2013).

[11] H. Zheng, J. A. Dijksman, and R. P. Behringer, Europhys. Lett. 107, 34005 (2014).

[12] D. Wang, J. Ren, J. A. Dijksman, H. Zheng, and R. P. Behringer, Phys. Rev. Lett. 120, 208004 (2018).

[13] M. Otsuki and H. Hayakawa, arXiv:1810.03846.

[14] S. Chen, T. Bertrand, W. Jin, M. D. Shattuck, and C. S. O’Hern, Phys. Rev. E 98, 042906 (2018).

[15] N. Kumar and S. Luding, Granular Matter 18, 58 (2016).

[16] T. Bertrand, R. P. Behringer, B. Chakraborty, C. S. O'Hern, and M. D. Shattuck, Phys. Rev. E 93, 012901 (2016).

[17] M. Baity-Jesi, C. P. Goodrich, A. J. Liu, S. R. Nagel, and J. P. Sethna, J. Stat. Phys. 167, 735 (2017).

[18] A. J. Liu and S. R. Nagel, Nature (London) 396, 21 (1998).

[19] M. E. Cates, J. P. Wittmer, J.-P. Bouchaud, and P. Claudin, Phys. Rev. Lett. 81, 1841 (1998).

[20] H. Vinutha and S. Sastry, Nat. Phys. 12, 578 (2016).

[21] S. Sarkar, D. Bi, J. Zhang, R. P. Behringer, and B. Chakraborty, Phys. Rev. Lett. 111, 068301 (2013).

[22] S. Sarkar and B. Chakraborty, Phys. Rev. E 91, 042201 (2015).

[23] S. Sarkar, D. Bi, J. Zhang, J. Ren, R. P. Behringer, and B. Chakraborty, Phys. Rev. E 93, 042901 (2016).
[24] R. P. Behringer and B. Chakraborty, Rep. Prog. Phys. 82, 012601 (2019).

[25] C. T. Veje, D. W. Howell, and R. P. Behringer, Phys. Rev. E 59, 739 (1999).

[26] J. Zhang, T. S. Majmudar, A. Tordesillas, and R. P. Behringer, Granular Matter 12, 159 (2010).

[27] R. Moosavi, M. R. Shaebani, M. Maleki, J. Török, D. E. Wolf, and W. Losert, Phys. Rev. Lett. 111, 148301 (2013).

[28] P. Coussot, J. S. Raynaud, F. Bertrand, P. Moucheront, J. P. Guilbaud, H. T. Huynh, S. Jarny, and D. Lesueur, Phys. Rev. Lett. 88, 218301 (2002).

[29] D. Fenistein and M. van Hecke, Nature (London) 425, 256 (2003).

[30] C. E. Maloney and A. Lemaître, Phys. Rev. E 74, 016118 (2006).

[31] See Supplemental Material at http://link.aps.org/ supplemental/10.1103/PhysRevLett.123.158001 for (1) more details of the setup, (2) a stress strain curve of a single particle, (3) the pressure calibration, (4) the fabric tensor calculation, (5) raw data for reverse shear tests, and (6) the choice of noise level, which includes Refs. [32,33].

[32] J. Barés, D. Wang, D. Wang, T. Bertrand, C. S. O'Hern, and R. P. Behringer, Phys. Rev. E 96, 052902 (2017).

[33] R. P. Behringer, D. Bi, B. Chakraborty, A. H. Clark, J. Dijksman, J. Ren, and J. Zhang, J. Stat. Mech. (2014) P06004.

[34] J. G. Puckett and K.E. Daniels, Phys. Rev. Lett. 110, 058001 (2013).

[35] Y. Zhao, J. Barés, H. Zheng, and R. P. Behringer, EPJ Web Conf. 140, 03049 (2017).

[36] K. E. Daniels, J. E. Kollmer, and J. G. Puckett, Rev. Sci. Instrum. 88, 051808 (2017).

[37] A. A. Zadeh, J. Barés, T. A. Brzinski, K. E. Daniels, J. Dijksman, N. Docquier, H. Everitt, J. E. Kollmer, O. Lantsoght, D. Wang, M. Workamp, Y. Zhao, and H. Zheng, Granular Matter 21, 83 (2019).

[38] L. Landau and E. Lifshitz, Course of Theoretical Physics (Pergamon Press, Oxford, 1986).

[39] Y. Zhao, H. Zheng, D. Wang, M. Wang, and R. P. Behringer, New J. Phys. 21, 023009 (2019).

[40] Y. Zhao, J. Barés, H. Zheng, C. S. Bester, Y. Xu, J. E. Socolar, and R.P. Behringer, Granular Matter 21, 90 (2019).

[41] T. S. Majmudar, M. Sperl, S. Luding, and R. P. Behringer, Phys. Rev. Lett. 98, 058001 (2007).

[42] C. S. O’Hern, L. E. Silbert, A. J. Liu, and S. R. Nagel, Phys. Rev. E 68, 011306 (2003).

[43] G. Debregeas, H. Tabuteau, and J.-M. di Meglio, Phys. Rev. Lett. 87, 178305 (2001).

[44] F. Varnik, L. Bocquet, J.-L. Barrat, and L. Berthier, Phys. Rev. Lett. 90, 095702 (2003).

[45] W. Losert, L. Bocquet, T. C. Lubensky, and J. P. Gollub, Phys. Rev. Lett. 85, 1428 (2000).

[46] P. Schall and M. van Hecke, Annu. Rev. Fluid Mech. 42, 67 (2010).

[47] L. E. Silbert, Soft Matter 6, 2918 (2010).

[48] M. van Hecke, J. Phys. Condens. Matter 22, 033101 (2010). 